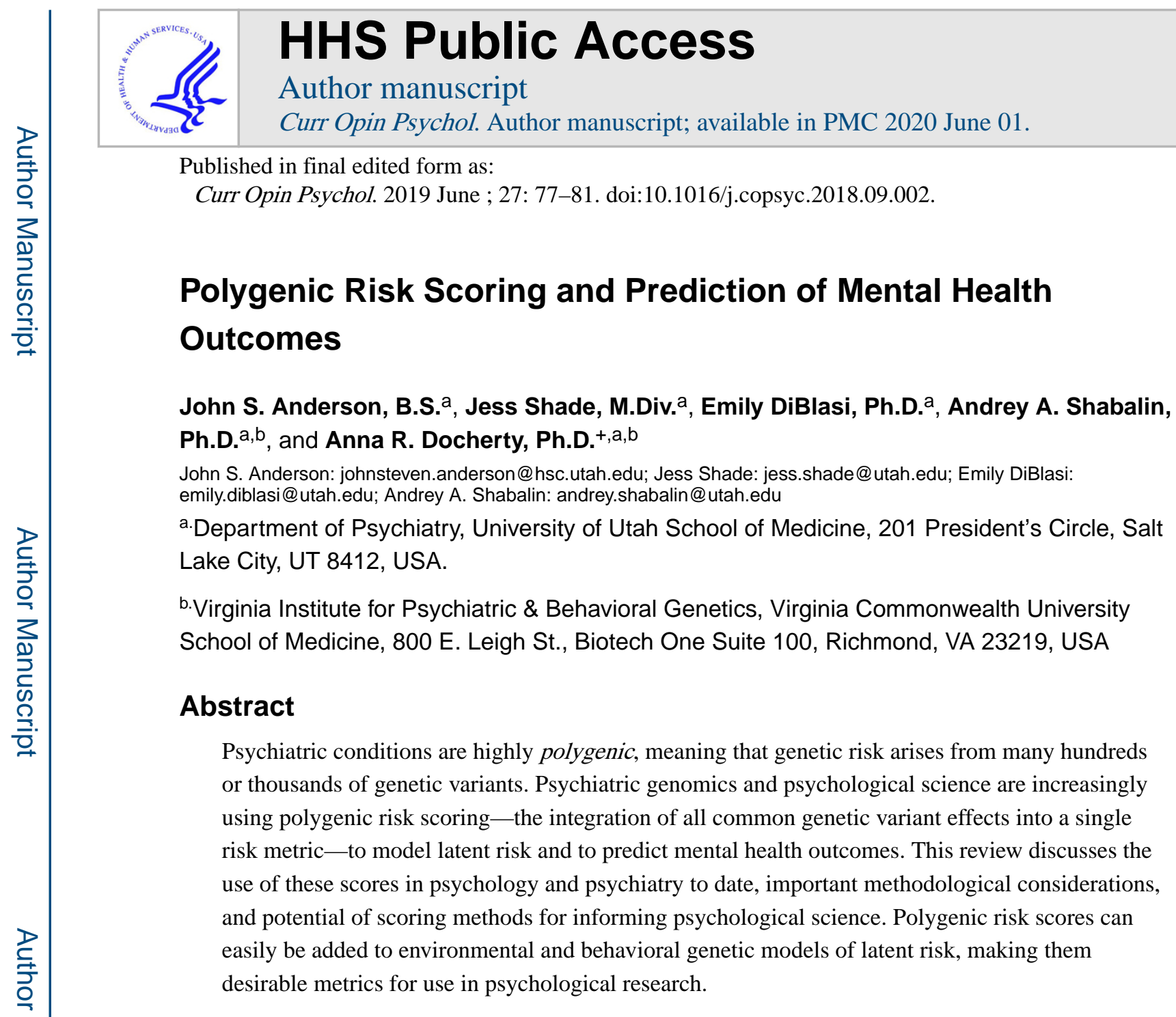

\title{
Introduction
}

Medical genetics has benefitted from gene discovery. For example, the discovery of BRCA mutations in determining lifetime risk of breast and ovarian cancers was revolutionary for prevention and treatment.[1],[2] While BRCA can predict the development of forms of breast cancer with high odds ratios,[1] we have no such biomarkers for most major psychiatric disorders. With the exception of family history, biomarkers have not yet proved to be reliable indicators of latent risk for these disorders.

\footnotetext{
${ }^{+}$Corresponding author, Department of Psychiatry, University of Utah School of Medicine, 501 Chipeta Way, Salt Lake City, Utah 84110, USA. tel. +1 801213 6905, fax. +1 804828 1471, anna.docherty@utah.edu.

Declaration of Interest:

Dr. Anna Docherty reports grants from National Institute of Mental Health (grant number K01MH093731), grants from Brain \& Behavior Research Foundation (NARSAD Young Investigator Award), and support from the University of Utah EDGE Scholar Program during the conduct of the review. The authors report no other conflicts of interest.

Publisher's Disclaimer: This is a PDF file of an unedited manuscript that has been accepted for publication. As a service to our customers we are providing this early version of the manuscript. The manuscript will undergo copyediting, typesetting, and review of the resulting proof before it is published in its final citable form. Please note that during the production process errors may be discovered which could affect the content, and all legal disclaimers that apply to the journal pertain.
} 
Twin and family studies have consistently demonstrated a strong genetic component to major psychiatric disorders, $[3,4]$ and molecular genetic studies have shown that psychiatric disorders are highly polygenic.[5] In the last decade, the field has reached consensus that, unlike many medical disorders associated with a single mutation of large effect (i.e. Mendelian), psychiatric disorders are actually associated with hundreds or thousands of genetic variants, each accounting for a small proportion of the variance in a phenotype.[6]

As a consequence, genome-wide association studies (GWAS) require massive sample sizes to detect even small effects on case status, and risk prediction in psychiatry and psychology has thus remained largely confined to phenotypic assessment. To address this problem, research in psychiatric genomics has moved toward statistical methods to integrate additive effects from across the entire genome into a single metric.

Just as phenotypic assessments to enhance prediction and early prevention have become broader in diagnostic scope, molecular genetic studies have expanded with the use of polygenic risk scores (PRS) to assess genetic risk for multiple phenotypes and to aggregate the effects of all common variants measured on the genome. With polygenic risk scoring, the rationale and goals of GWAS have been extended beyond simply finding "hits" on specific locations of the genome to providing a continuous metric for powerful polygenic prediction of complex traits like schizophrenia and major depression.[7] By aggregating all variant effects into one continuous metric, one can also skirt the strict multiple comparisons corrections necessary for GWAS. In other words, rather than correcting for millions of univariate statistical tests looking for hits across the genome, just one statistical test is required to regress genome-wide PRS onto the phenotype of interest. [8]

It is increasingly apparent that psychiatric genomics will be instrumental to improving clinical screening and prediction efforts, but genomic modeling of psychiatric risk is complex, and it is now broadly accepted that in most cases genetic modeling must entail close examination of the entire genome. In the context of examining the effects of environmental exposures on outcomes of interest, models incorporating both polygenic risk and environmental risk metrics are relatively straightforward. Below, we briefly review common methods for scoring polygenic risk, the use of PRS to date, and their general potential to move psychological science forward.

\section{Polygenic Risk Scores}

Genome-wide polygenic risk scoring is also referred to as polygenic profile scoring, genetic profile scoring, or genotype scoring. In this type of analysis, all genome-wide common variants, measured or imputed, can be incorporated into a single score used for the prediction of a trait and for association with outcomes. Each variant in the test sample is weighted according to the variant's effect size in the training sample, the genome-wide association study.

In other words, to calculate polygenic risk scores (PRS), summary statistics from previous GWAS (with at least ten thousand cases and controls) are used as the "discovery" statistics for the trait of interest. In such GWAS, referred to as the training GWAS or training sample,

Curr Opin Psychol. Author manuscript; available in PMC 2020 June 01. 
allele frequencies for each common variant are tested for association with the outcome phenotype of interest (e.g., schizophrenia case-control status, or trait impulsivity). Summary statistics from these training GWAS include an effect size (frequently reported as an odds ratio) and a p-value for each SNP tested. Details of how these statistics are used to calculate PRS can be found in Box 1.

Training GWAS with larger sample sizes are more likely to detect subtle genetic variation in polygenic traits and can provide more precise estimates of effect sizes. The PRS calculated using summary statistics from larger GWAS are likely to have lower error and improved predictive properties, i.e. higher correlation with the phenotype of interest in the new (independent) sample. Thus the $\mathrm{N}$ of the training sample is critical to the success of PRS as a predictor. Large GWAS and GWAS meta-analyses for most psychiatric disorders have been conducted, and the resulting GWAS summary statistics for PRS calculation in independent data sets are readily available online.

A principal advantage of using a polygenic risk scoring approach is that it estimates an individual's genome-wide risk for a disorder or trait, aggregating all common variants. Because all common variants are included in PRS calculation additively, the genome-wide metric is typically normally distributed in the population and can be used in much the same way that common assessment scores (e.g., a depression inventory) are used in statistical models of prediction. Again, this allows for well-powered analyses of the sample sizes typical of most psychological labs $(\mathrm{N}<300)$ prioritizing the collection of rich, nuanced phenotypic data.

Not only does the metric make genetic data more computationally manageable for projects in psychological science, but there are already established PRS software[11,12] and readily available discovery GWAS summary statistics from collaborative groups such as the Psychiatric Genomics Consortium. For these reasons, PRS is a very convenient metric for use in psychological research.

\section{Prediction of Outcomes and Enhancement of Nosology}

Although in its infancy, polygenic risk scoring can already predict discrete mental health outcomes with some success in case-control samples.[13] For example, PRS can predict case status relating to psychosis spectrum disorders,[14] mania,[15] major depression,[16] and ADHD[17]. It is important to note that as of 2018, however, prediction of case-control status is confined to studies of case-control cohorts, and is not yet applicable to most DSM diagnoses in population-based samples.

The lower the base rate for a disorder, the less powerful a prediction method will be in the general population. The recent development of new strategies for making PRS-based predictions, including structural equation modeling of genome-wide data and multipolygenic scoring, will likely further increase the predictive utility of PRS.[18,19]

Looking beyond narrow definitions of psychiatric diagnosis, PRS has already been successful in 1) the prediction of symptom presentation in clinical samples, and 2) the 
prediction of risk traits in healthy individuals. Both areas are expanding rapidly and findings are consistent with dimensional conceptualizations of psychopathology.

One prime example of the first area is research on psychosis-spectrum psychopathology: psychosis is quite heritable and polygenic, thus a recently demonstrated PRS association with diagnosis in case-control samples is not surprising.[20] But within psychosis-spectrum cases, bipolar disorder PRS is significantly predictive of manic symptom presentation.[15] Conversely, schizophrenia PRS are strongly related to the level of mood-incongruent psychotic symptoms in bipolar disorder.[21] Both schizophrenia and bipolar PRS predict family history in the proband, predict prognosis,[22] have also predicted addiction-related outcomes in a study of 144,000 individuals with a wide range of addiction-related impairment.[23]

In addition to predicting symptom presentation, PRS are also predictive of psychiatric and health outcomes in otherwise healthy individuals. In a general population of college students, PRS for schizophrenia and neuroticism have predicted anxiety, depressive symptoms, family history of psychiatric disorders, and smoking behavior.[24] In a population-based study of UK adolescents, PRS were strongly associated with interest and self-efficacy in math and sciences, as well as behavioral and conduct problems.[25] High PRS for bipolar disorder increases risk for the development of PTSD in military veterans. [26] The application to outcomes is wide-ranging: neurocognitive ability,[27] and even creativity [28] have been associated with PRS for schizophrenia. These findings suggest that PRS for many disorders can be applied across populations, diagnoses, traits, and symptom dimensions to inform risk prediction.

PRS can be associated with markers of well-being and resilience as well. For example, educational attainment [29] is predicted by PRS, as are both subjective well-being and extraversion. In healthy individuals, resilience-related PRS are additionally associated with decreased anxiety, neuroticism, depressive symptoms, and smoking behavior, and with increased well-being and extraversion.[24]

PRS may be leveraged to identify different latent categories of risk and resilience, to explore physiological biomarkers of mental health,[30] and to inform research on new gene pathways or drug targets.[31] In psychological science, it is hoped this may enhance nosology by adding new layer of information to the multi-trait, mutli-method approach, thereby refining phenotypic measures of latent risk.

\section{Limitations of Polygenic Risk Scoring}

There are methodological considerations to make explicit in any discussion of PRS, and we will briefly review three very important points here. First, current $P R S$ cannot be used in cohorts of non-European ancestry unless the discovery GWAS is of the same ancestry. Because large GWAS of other ancestry groups are scarce, PRS analyses are currently limited to only cohorts of European ancestry. [32,33] Lack of signal from European PRS across ancestry, within a U.S. cohort, has already been reported.[34] As ethnic minority populations already experience considerable health care disparities, the limitation of utilizing PRS 
prediction strategies to European ancestry serves to further add to disparities, and demands attention from the research community. Sample sizes required for appropriately-powered discovery GWAS require significant resources[35] but work from the Psychiatric Genomics Consortium is addressing this concern.[36]

Second, PRS will always be limited by the sensitivity and specificity of the trait it reflects, and consequently the shortcomings inherent to our current diagnostic system. One way to address this concern would be to begin to map PRS to a variety of measures and methods to help inform nosology. One can now create PRS for several hundred psychiatric and medical conditions, and apply them to a single cohort of interest.

Third, simulations demonstrate that liberal PRS p-value thresholds (i.e. those which include the majority of the genome in their calculation) improve predictive utility, yet such a strategy could produce overly-conservative estimates in certain conditions.[9] In the absence of an a priori optimal threshold, one may consider reading the formal analysis of thresholding recently published online.[37] In current practice, it is common to calculate PRS at a variety of SNP association thresholds and examine a plot of the variance accounted for in the trait across thresholds. Many studies have selected the best-performing threshold after subsequent analyses are performed. However, any "cherry-picking" of PRS p-value thresholds conflates signal, reducing replicability across studies. Accounting for multiple testing is imperative, and empirically-validated strategies for a priori selection of thresholds for PRS are still in development.

\section{Conclusions}

PRS methods have unlocked complex traits, making them accessible for genetic study and clinical evaluation in a way that was impossible a decade ago. As polygenic risk scoring continues to revolutionize the scope of psychiatric and behavioral genetics, the use of PRS in psychological science will grow exponentially.[34,38]

It is clear that PRS methods are powerful and are not going away. Polygenic risk scoring comes at a time when the mental health field increasingly questions categorical diagnostic approaches. PRS are particularly in step with the movement of the field toward understanding psychiatric disorders as latent categories and continuous dimensions, with increasing support for the latter.[39] Indeed, genome-wide strategies have already been useful in mapping genetic overlap of major disorders, but will likely continue to provide important information to inform the hierarchical taxonomy of symptom dimensions.[40,41]

Furthermore, PRS allows for the measurement of risk of psychiatric and medical disorders simultaneously. For example, depressed individuals who carry high polygenic risk of elevated triglycerides or BMI may require different treatment approaches to those without such risk. GWAS have isolated genetic associations with treatment response and side effect profiles,[22,42] and as such efforts continue, PRS may also help to predict drug and psychotherapy treatment response.

PRS will likely never be a replacement for well-trained clinical expertise, but may serve to supplement clinical knowledge as we consider the prediction of severe psychopathology in 
the general population. By leveraging PRS in symphony with family history, electronic medical record data, and clinical assessments, those in need of specific intervention may be identified earlier and more reliably. Ideally, advancements in machine learning and structural equation modeling will help to orchestrate these components.[43] Such strategies are proving successful in other areas of medicine[44-46] and are necessary for psychiatry and psychological science moving forward.

\section{Acknowledgements}

Funding: This work was supported by the National Institute of Mental Health (grant number K01MH093731), the Brain \& Behavior Research Foundation, and the University of Utah EDGE Scholar Program.

\section{Cited References}

[1]. Miki Y, Swensen J, Shattuck-Eidens D, Futreal PA, Harshman K, Tavtigian S, Liu Q, Cochran C, Bennett LM, Ding W, et al.: A strong candidate for the breast and ovarian cancer susceptibility gene BRCA1. Science 1994, 266:66-71. [PubMed: 7545954]

[2]. Manchanda R, Legood R, Burnell M, McGuire A, Raikou M, Loggenberg K, Wardle J, Sanderson $\mathrm{S}$, Gessler S, Side L, et al.: Cost-effectiveness of population screening for BRCA mutations in Ashkenazi jewish women compared with family history-based testing. J Natl Cancer Inst 2014, 107:dju380. [PubMed: 25435542]

[3]. Cardno AG, Marshall EJ, Coid B, Macdonald AM, Ribchester TR, Davies NJ, Venturi P, Jones LA, Lewis SW, Sham PC, et al.: Heritability estimates for psychotic disorders: the Maudsley twin psychosis series. Arch Gen Psychiatry 1999, 56:162-168. [PubMed: 10025441]

[4]. McGue M, Christensen K: The heritability of depression symptoms in elderly Danish twins: occasion-specific versus general effects. Behav Genet 2003, 33:83-93. [PubMed: 14574144]

[5]. Sullivan PF, Daly MJ, O'donovan M: Genetic architectures of psychiatric disorders: the emerging picture and its implications. Nat Rev Genet 2012, 13:537. [PubMed: 22777127]

[6]. Gratten J, Wray NR, Keller MC, Visscher PM: Large-scale genomics unveils the genetic architecture of psychiatric disorders. Nat neurosci 2014, 17:782. [PubMed: 24866044]

[7]. Visscher PM, Brown MA, McCarthy MI, Yang J: Five years of GWAS discovery. Am J Hum Genet 2012, 90:7-24. [PubMed: 22243964]

[8]. Wray NR, Goddard ME, Visscher PM: Prediction of individual genetic risk to disease from genome-wide association studies. Genome res 2007, 17:1520-1528. [PubMed: 17785532]

[9]. Dudbridge F: Power and predictive accuracy of polygenic risk scores. PLoS genet 2013, 9:e1003348. [PubMed: 23555274]

[10]. International Schizotypy Consortium: Common polygenic variation contributes to risk of schizophrenia and bipolar disorder. Nature 2009, 460:748. [PubMed: 19571811]

[11]. Vilhjálmsson BJ, Yang J, Finucane HK, Gusev A, Lindström S, Ripke S, Genovese G, Loh P-R, Bhatia G, Do R, et al.: Modeling linkage disequilibrium increases accuracy of polygenic risk scores. Am J Hum Genet 2015, 97:576-592. [PubMed: 26430803]

[12]. Euesden J, Lewis CM, O'Reilly PF: PRSice: polygenic risk score software. Bioinformatics 2014, 31:1466-1468. [PubMed: 25550326]

[13]. Wray NR, Lee SH, Mehta D, Vinkhuyzen AA, Dudbridge F, Middeldorp CM: Research review: polygenic methods and their application to psychiatric traits. J Child Psychol Psychiatry 2014, 55:1068-1087. [PubMed: 25132410]

[14]. Tesli M, Espeseth T, Bettella F, Mattingsdal M, Aas M, Melle I, Djurovic S, Andreassen O: Polygenic risk score and the psychosis continuum model. Acta Psychiatr Scand 2014, 130:311317. [PubMed: 24961959]

[15]. Ruderfer DM, Fanous AH, Ripke S, McQuillin A, Amdur RL, Schizophrenia Working Group of the Pyschiatic Genomics Consortium, Bipolar Disorder Working Group of the Pyschiatric Genomics Consortium, Cross-disorder Working Group of the Pyshciatric Genomics Consortium, 
Gejman PV, O'Donovan MC, et al.: Polygenic dissection of diagnosis and clinical dimensions of bipolar disorder and schizophrenia. Mol Psychiatry 2014, 19:1017. [PubMed: 24280982]

[16]. Genomics of Personality Consortium: Meta-analysis of genome-wide association studies for neuroticism, and the polygenic association with major depressive disorder. JAMA psychiatry 2015, 72:642-650. [PubMed: 25993607]

[17]. Groen-Blokhuis MM, Middeldorp CM, Kan K-J, Abdellaoui A, van Beijsterveldt CE, Ehli EA, Davies GE, Scheet PA, Xiao X, Hudziak JJ, et al.: Attention-deficit/hyperactivity disorder polygenic risk scores predict attention problems in a population-based sample of children. $\mathrm{J} \mathrm{Am}$ Acad Child Adolesc Psychiatry 2014, 53:1123-1129. e1126. [PubMed: 25245356]

[18]. Maier R, Moser G, Chen G-B, Ripke S, Absher D, Agartz I, Akil H, Amin F, Andreassen OA, Anjorin A: Joint analysis of psychiatric disorders increases accuracy of risk prediction for schizophrenia, bipolar disorder, and major depressive disorder. Am J Hum Genet 2015, 96:283294. [PubMed: 25640677]

*[19]. Krapohl E, Patel H, Newhouse S, Curtis CJ, von Stumm S, Dale P, Zabaneh D, Breen G, O'reilly P, Plomin R: Multi-polygenic score approach to trait prediction. Mol Psychiatry 2017.By using cross-validation to select from and estimate contributions of of 81 different PRS on traits of interest, the authors were able to significantly increase predictive ability of their model compared to prediction of the traits with just a single polygenic risk score. This study demonstrates that innovative polygenic risk approaches are apt to increase preditive ability of these scores moving forward.

[20]. Cardno AG, Rijsdijk FV, Sham PC, Murray RM, McGuffin P: A twin study of genetic relationships between psychotic symptoms. Am J Psychiatry 2002, 159:539-545. [PubMed: 11925290]

[21]. Allardyce J, Leonenko G, Hamshere M, Pardiñas AF, Forty L, Knott S, Gordon-Smith K, Porteous DJ, Haywood C, Di Florio A, et al.: Association Between Schizophrenia-Related Polygenic Liability and the Occurrence and Level of Mood-Incongruent Psychotic Symptoms in Bipolar Disorder. JAMA Psychiatry 2018, 75:28-35. [PubMed: 29167880]

[22]. Adkins DE, Åberg K, McClay JL, Bukszár J, Zhao Z, Jia P, Stroup TS, Perkins D, McEvoy JP, Lieberman JA, et al.: Genomewide pharmacogenomic study of metabolic side effects to antipsychotic drugs. Mol Psychiatry 2011, 16:321. [PubMed: 20195266]

[23]. Reginsson GW, Ingason A, Euesden J, Bjornsdottir G, Olafsson S, Sigurdsson E, Oskarsson H, Tyrfingsson T, Runarsdottir V, Hansdottir I, et al.: Polygenic risk scores for schizophrenia and bipolar disorder associate with addiction. Addict Biol 2018, 23:485-492. [PubMed: 28231610]

[24]. Docherty AR, Moscati A, Dick D, Savage JE, Salvatore JE, Cooke M, Aliev F, Moore AA, Edwards AC, Riley BP, et al.: Polygenic prediction of the phenome, across ancestry, in emerging adulthood. Psychol Med 2017:1-10.

*[25]. Krapohl E, Euesden J, Zabaneh D, Pingault J, Rimfeld K, Von Stumm S, Dale P, Breen G, O'reilly P, Plomin R: Phenome-wide analysis of genome-wide polygenic scores. Mol Psychiatry 2016, 21:1188. [PubMed: 26303664] This study of UK adolescents predicted a variety of cognitive and psychiatric traits with PRS. Notably, they demonstrated that stratefying the adolescents into septiles of PRS yeiled relatively large separation for the traits of interest. This research shows how PRS can be used for informative screening in the general population.

[26]. Nievergelt CM, Maihofer AX, Mustapic M, Yurgil KA, Schork NJ, Miller MW, Logue MW, Geyer MA, Risbrough VB, O’Connor DT, Baker DG: Genomic predictors of combat stress vulnerability and resilience in US Marines: a genome-wide association study across multiple ancestries implicates PRTFDC1 as a potential PTSD gene. Psychoneuroendocrinology 2015, 51:459-471. [PubMed: 25456346]

[27]. Lencz T, Knowles E, Davies G, Guha S, Liewald DC, Starr JM, Djurovic S, Melle I, Sundet K, Christoforou A, et al.: Molecular genetic evidence for overlap between general cognitive ability and risk for schizophrenia: a report from the Cognitive Genomics consorTium (COGENT). Mol Psychiatry 2014, 19:168. [PubMed: 24342994]

[28]. Power RA, Steinberg S, Bjornsdottir G, Rietveld CA, Abdellaoui A, Nivard MM, Johannesson M, Galesloot TE, Hottenga JJ, Willemsen G, et al.: Polygenic risk scores for schizophrenia and bipolar disorder predict creativity. Nat Neurosci 2015, 18:953-955. [PubMed: 26053403] 
**[29]. Selzam S, Krapohl E, von Stumm S, O'reilly P, Rimfeld K, Kovas Y, Dale PS, Lee J, Plomin R: Predicting educational achievement from DNA. Mol Psychiatry 2017, 22:267. [PubMed: 27431296] In this publication, the authors demonstrate PRS for educational attainment accounts for more variance than GWAS, and that PRS predicted 15\% of the variance for education acheivement. Because these PRS were also associated with other factors impacting educational attainment like socioeconomic status and cognitive ability, this study is a powerful demonstration of how PRS can be leveraged in the social sciences to predict risk and resilience.

[30]. Sabuncu MR, Buckner RL, Smoller JW, Lee PH, Fischl B, Sperling RA, Alzheimer;s Disease Neuroimaging Initiative: The association between a polygenic Alzheimer score and cortical thickness in clinically normal subjects. Cereb Cortex 2011, 22:2653-2661. [PubMed: 22169231]

[31]. Schizophrenia Working Group of the Pyschiatric Genomics Consortium: Biological insights from 108 schizophrenia-associated genetic loci. Nature 2014, 511:421. [PubMed: 25056061]

[32]. Hilton CL, Fitzgerald RT, Jackson KM, Maxim RA, Bosworth CC, Shattuck PT, Geschwind DH, Constantino JN: Brief report: Under-representation of African Americans in autism genetic research: A rationale for inclusion of subjects representing diverse family structures. J Autism Dev Disord 2010, 40:633-639. [PubMed: 19936905]

[33]. Burchard EG, Oh SS, Foreman MG, Celedón JC: Moving toward true inclusion of racial/ethnic minorities in federally funded studies. A key step for achieving respiratory health equality in the United States. Am J Respir Crit Care Med 2015, 191:514-521. [PubMed: 25584658]

[34]. Torkamani A, Wineinger NE, Topol EJ: The personal and clinical utility of polygenic risk scores. Nat Rev Genet 2018:1.

[35]. Spencer CC, Su Z, Donnelly P, Marchini J: Designing genome-wide association studies: sample size, power, imputation, and the choice of genotyping chip. PLoS genet 2009, 5:e1000477. [PubMed: 19492015]

[36]. Sullivan PF: The psychiatric GWAS consortium: big science comes to psychiatry. Neuron 2010, 68:182-186. [PubMed: 20955924]

[37]. Ware EB, Schmitz LL, Faul JD, Gard A, Mitchell C, Smith JA, Zhao W, Weir D, Kardia SLR: Heterogeneity in polygenic scores for common human traits. bioRxiv 2017.

*[38]. Lewis CM, Vassos E: Prospects for using risk scores in polygenic medicine. Genome Med 2017, 9:96. [PubMed: 29132412]

[39]. Kotov R, Krueger RF, Watson D, Achenbach TM, Althoff RR, Bagby RM, Brown TA, Carpenter WT, Caspi A, Clark LA, et al.: The Hierarchical Taxonomy of Psychopathology (HiTOP): A dimensional alternative to traditional nosologies. J Abnorm Psychol 2017, 126:454. [PubMed: 28333488] In an attempt to address the limitations of arbitrary boundaries between disorders, diagnostic instability, and disorder heterogenity of the DSM, the authors outline a dimenional structure of pyschopathology. Disorders are grouped into emprically-derived subfactors, spectra, and super-spectra. This paradigm shift is better modeled by polygenic risk scoring methods than traditional nosology and better reflects latent liability for psychopathology.

[40]. Cross-Disorder Group of the Psychiatric Genomics Consortium: Identification of risk loci with shared effects on five major psychiatric disorders: a genome-wide analysis. Lancet 2013, 381:1371-1379. [PubMed: 23453885]

[41]. Bulik-Sullivan B, Finucane HK, Anttila V, Gusev A, Day FR, Loh P-R, ReproGen Consortium, Pyschiatric Genomics Consortium, Genetic Consortium for Anorexia Nervosa of the Wellcome Trust Case Control Consortium 3, Duncan L, et al.: An atlas of genetic correlations across human diseases and traits. Nat Genet 2015, 47:1236. [PubMed: 26414676]

[42]. Frank J, Lang M, Witt S, Strohmaier J, Rujescu D, Cichon S, Degenhardt F, Nöthen M, Collier D, Ripke $S$, et al.: Identification of increased genetic risk scores for schizophrenia in treatmentresistant patients. Mol Psychiatry 2015, 20:150. [PubMed: 24888364]

*[43]. Paré G, Mao S, Deng WQ: A machine-learning heuristic to improve gene score prediction of polygenic traits. Sci Rep 2017, 7:12665. [PubMed: 28979001] The machine-learning heuristic in this study significantly outperformed exsiting polygenic risk scoring methods, and required a relatively small sample for calibration. Methods such as these could be instrumental to making PRS powerful enough for clinical applications. 
[44]. Aly M, Wiklund F, Xu J, Isaacs WB, Eklund M, D'Amato M, Adolfsson J, Grönberg H: Polygenic risk score improves prostate cancer risk prediction: results from the Stockholm-1 cohort study. Eur Urol 2011, 60:21-28. [PubMed: 21295399]

[45]. Meigs JB, Shrader P, Sullivan LM, McAteer JB, Fox CS, Dupuis J, Manning AK, Florez JC, Wilson PW, D'Agostino RB Sr, Cupples LA: Genotype score in addition to common risk factors for prediction of type 2 diabetes. N Engl J Med 2008, 359:2208-2219. [PubMed: 19020323]

[46]. Pharoah PD, Antoniou AC, Easton DF, Ponder BA: Polygenes, risk prediction, and targeted prevention of breast cancer. N Engl J Med 2008, 358:2796-2803. [PubMed: 18579814] 


\section{Box 1: Calculating Polygenic Risk Scores}

Briefly, PRS is traditionally calculated as:

$$
\mathrm{PRS}_{k}=\sum_{i} \beta_{i} \mathrm{SNP}_{i k}
$$

where PRS for individual $k$ in the target sample is calculated by the summation of each SNP (measured for both the person $k$ and passing a set association threshold in the discovery GWAS) multiplied by the effect size, $\beta$, of that SNP in the discovery GWAS. [8] Discovery GWAS association thresholds required for inclusion in the PRS calculation can be set at any p-value, but are often set closer to 1.0 to incorporate more of the genome.[9] There are also variations on the traditional method developed by Purcell et al.,[10] Bayesian and otherwise,[11] (T Mak et al., unpublished) which are not reviewed here. 